up of experts in each eligible condition. Data fields include demographics, blood and urine results, medications, transplant and dialysis history, genetics and co-morbidities. Data is entered retrospectively from the patient's medical records following consent.

Results 319 patients have been consented at $\mathrm{BCH}$. The age range is from birth to 16 years with mean of 4.9 years with male to female ratio of $55 \%: 45 \%$. The most common condition is Idiopathic Nephrotic Syndrome $(n=128 ; 39 \%)$, followed by Alport Syndrome $(n=33 ; 10 \%)$, ARPKD $(n=25$; $8 \%)$, Hyperoxaluria $(n=24 ; 7 \%)$ and STEC HUS $(n=22 ; 7 \%)$. The other conditions with numbers of patients recruited so far include: ADPKD $(n=14)$, aHUS $(n=13)$; Cystinosis $(n=9)$; Cystinuria $(n=3)$; Dent and Lowe $(n=7)$; HNF1b $(n=6)$; Hypokalaemic Alkalosis $(n=8)$; MPGN $(n=14)$ and Vasculitis $(n=7)$.

Conclusion $\mathrm{RaDaR}$ provides important epidemiology data based on the whole country population which is shared amongst the renal team to develop further research into rare kidney diseases and improve the quality of care for these patients. It also gives an opportunity to define the best treatment practices across the country in the future.

\section{G34(P) ABSTRACT WITHDRAWN}

\section{G35(P) ROLE OF LUMBAR PUNCTURE IN INFANTS PRESENTING WITH RECURRENT APNOEA}

R Manuel, P Ramesh. Paediatric Intensive Care Unit, University Hospital of North Midlands NHS Trust, Stoke on Trent, UK

\subsection{6/archdischild-2018-rcpch.33}

Background The commonest cause of recurrent apnoea in early infancy is due to bronchiolitis especially when they present with other respiratory symptoms. Lumbar puncture is commonly performed in these babies to rule out the possibility of meningitis. Almost all these babies are also commenced on antibiotics and antiviral agents to cover meningitis until it is excluded.

Aim To identify the incidence of meningitis in infants presenting with recurrent apnoea to a tertiary paediatric intensive care unit.

Methods Prospective collection of data and retrospective review of case notes of all the infants intubated and ventilated because of recurrent apnoea. The study period was for three and a half years from October 2014 to February 2017.

Results Sixty five infants were included in the study. Their mean age was 5 weeks (range: 3 weeks to 3 months). All of them had a non-bronchoscopic Broncho Alveolar Lavage (BAL) to identify respiratory pathogens and also had lumbar puncture to rule out meningitis. All the infants presented with a history of coryzal symptoms, poor feeding, respiratory distress and recurrent apnoea. The average highest peak CRP for this group was 73 (interquartile range: 26-103). 39 babies $(60 \%)$ received fluid resuscitation at presentation.

PCR test was positive in the BAL for a viral pathogen in sixty infants (92\%) and negative in five (8\%). Two babies in the
BAL positive group also had a positive PCR test for a virus in the CSF. One baby had enterovirus positive in both BAL and CSF and another baby was PCR positive for Rhinovirus in BAL and HHV6 in CSF. The CSF cell count was normal in both these infants. No infant had evidence of bacterial meningitis or a treatable cause of viral meningitis.

Conclusion Our results show that there is a low incidence of possible viral meningitis in infants presenting with recurrent apnoea and other respiratory symptoms. None of the babies would have benefitted from antibiotics or antiviral agents.

\section{G36(P) OUTCOMES OF INFANTS TRANSFERRED FROM THE NEONATAL INTENSIVE CARE TO THE PAEDIATRIC WARD AND PAEDIATRIC INTENSIVE CARE AFTER 44 WEEKS CORRECTED GESTATIONAL AGE}

\begin{abstract}
${ }^{1,2} \mathrm{~K}$ Crawford, 'SZ Kamupira, 'S Morley, ${ }^{2} \mathrm{AW}$ Kelsall. ${ }^{1}$ Paediatric Intensive Care, Cambridge University NHS Foundation Trust, Cambridge, UK; ${ }^{2}$ Neonatology, Cambridge University NHS Foundation Trust, Cambridge, UK
\end{abstract}

10.1136/archdischild-2018-rcpch.34

Aim NHS England has outlined the Neonatal Intensive Care Unit (NICU) service to be for infants who are generally (but not exclusively) $<44$ weeks corrected gestational age (CGA). There are implications for the Paediatric Intensive Care Unit (PICU) and general paediatric wards in terms of managing this workload, and transitioning complex infants. Following challenges in accommodating some of these cases within our services, we conducted a study to assess the workload and outcomes of infants transferred from NICU after 44 weeks CGA over a 5 year period.

Methods Eligible infants transferred from NICU between 1/09/ 2010 - 31/08/2015 were identified from the Standardised Electronic Neonatal Database (SEND) and PICU databases. Information was extracted from the SEND discharge summaries and medical records regarding transition of care.

Results 26 infants were identified. A mean of 4 (1-6) infants were transitioned annually to PICU or the paediatric wards. Median birth gestation was $36+{ }^{2}\left(23+{ }^{6}-41+{ }^{1}\right)$ weeks, weight of $2400(515-4480)$ grams. Median transition age was $46+{ }^{1}$ $\left(44+{ }^{0}-52+{ }^{6}\right)$ weeks, weight of $3417(1890-6170)$ grams. 11 of the 26 infants identified were transferred from NICU to PICU. Median length of stay (LOS) on PICU was 28 (1-241) days, mean 47 days. PICU occupancy per annum was 517 days, $13 \%$ of the annual bed occupancy (3754 days). 22 infants were transferred to the general paediatric wards, including some of whom were initially on PICU. Median LOS on the general paediatric ward was 11 (1-145) days, total of 591 days annually. No child died before discharge from hospital.

Conclusion This small group of complex infants constitute a high workload for both PICU and the general paediatric wards. The majority of infants remaining in hospital post 44 weeks CGA had undergone surgery during the neonatal period. The knowledge obtained from this study will help in planning the timely transition of these infants to paediatric services. This may help prevent local capacity issues to paediatric services by neonates awaiting repatriation around the region and familial separation. 